\title{
NOTAS SOBRE LA ANALOGÍA ENTRE LOS SISTEMAS DE MEDIDA DE LOS OBJETOS FÍSICOS Y LOS ESTADOS MENTALES DE LOS SERES HUMANOS
}

DIANA I. PÉREZ

CONICET

UNIVERSIDAD DE BUENOS AIRES

Los estados mentales a los que se alude con la expresión "actitudes proposicionales" suelen ser considerados estados en los que se establece una relación (de creencia, deseo, temor, etc.) entre un individuo y una proposición, es decir, una entidad abstracta. Así, se suele sostener que cuando se afirma "Juan cree que la luna es redonda" se está afirmando que entre el individuo nombrado por la expresión "Juan" y la proposición expresada por la oración "la luna es redonda" se da una relación de creencia. Lo mismo ocurre, mutatis mutandis, con afirmaciones del tipo "María desea que este día tenga 25 horas", "José teme que su esposa se haya enterado de la verdad", etc. También es ampliamente aceptada hoy en día la idea de que deseos y creencias, conjuntamente, causan las acciones humanas. Sin embargo, resulta complicado entender cómo es que la relación de un individuo con una entidad abstracta como es una proposición pueda ser la causa de evento alguno. ${ }^{1}$ Para hacer comprensible esta relación causal varios autores han apelado a la analogía existente entre las actitudes proposicionales y su poder para causar acciones, por un lado, y lo que se ha dado en llamar la "actitud numérica" de los objetos físicos y su poder para causar eventos físicos, por el otro. Parece no haber inconvenientes para sostener que, aunque el peso de un cuerpo pueda entenderse como la relación existente entre un objeto físico y cierto número (el que utilizamos para expresar su peso en kilogramos, por ejemplo), es esta relación entre el objeto físico y cierta entidad abstracta —l número en cuestión- la que causa efectivamente ciertos cambios en nuestro mundo. Así, por ejemplo, afirmamos que fue el peso de cinco kilogramos de este objeto lo que causó que se rompiera la mesa de vidrio sobre la que estaba apoyado. ${ }^{2}$

1 En este trabajo, llamaremos a este problema, el problema de la causación mental.

2 Algunos de los trabajos en los que se ha intentado explotar esta analogía, aunque no siempre con la misma finalidad, son: Churchland (1984) Matter and Consciousness, MIr Press, 
Donald Davidson, por su parte, también recoge la analogía entre las actitudes proposicionales y las actitudes numéricas. ${ }^{3}$ En el presente trabajo intentaré mostrar cómo esta analogía permite echar luz sobre ciertas tesis davidsonianas ampliamente controvertidas acerca de la naturaleza de lo mental, extendiéndose más allá del problema de la causación mental. Para ello expondré paso a paso la analogía señalando una serie de similitudes que se pueden encontrar entre nuestro hablar acerca de la medida de los objetos físicos macroscópicos, y nuestro hablar acerca de la vida mental ${ }^{4}$ de los seres humanos. Por un lado, mostraré en qué medida la analogía mencionada ayuda a incorporar dentro de la teoría davidsoniana de lo mental - su monismo anómalo- la tesis de la inexistencia de "objetos ante la mente" y la aceptación de la tesis externalista acerca del contenido mental (apartados [a]-[d]). Por otro lado, me detendré en la utilización que Davidson hace de esta analogía en "Mental Events" para especificar el papel que desempeña la racionalidad en su teoría de lo mental. Mostraré que dicha analogía permite comprender el papel que Davidson asigna a la racionalidad, pero nos da pocas pistas para especificar en qué consiste tal racionalidad (apartado [e]). Por último, mencionaré brevemente la respuesta que propone Davidson al problema que popularizó esta analogía en la literatura filosófica de los últimos años: el problema de la causación mental (apartado [f]). ${ }^{5}$

Veamos entonces con cierto detalle en qué consiste exactamente la analogía propuesta entre los sistemas de medición de objetos físicos macroscópicos, y los sistemas utilizados para adscribir estados mentales a los seres humanos. A primera vista, es posible señalar una semejanza estructural entre (i) las oraciones por medio de las cuales se atribuye una medida a un cuerpo físico y (ii) las oraciones por medio de las cuales se atribuye una actitud proposicional a un ser humano. Por ejemplo, sean:

caps. 3 y 4; Stalnaker (1984) Inquiry, Mrr Press; Dennett (1980) "Beyond Belief", reimpreso en Dennett (1987) The Intentional Stance, Mrr Press, cap. 5; además de los trabajos de Davidson enlistados en la n. 3.

3 Davidson apela a esta analogía en varios artículos, a saber: Davidson (1977) "Reality without Reference", reimpreso en Davidson (1984) Inquiries into Truth and Interpretation, Oxford University Press; Davidson (1970) "Mental Events", reimpreso en Davidson (1980) Essays on Actions and Events, Oxford University Press; y Davidson (1991) "What is Present to the Mind?", en Philosophical Issues, no. 1 [citado siguiendo la traducción castellana de Olbeth Hansberg "¿Qué está presente ante la mente?", en Valdés (comp.) (1996) Pensamiento y lenguaje. Problemas en la atribución de actitudes proposicionales, México, UNAM].

4 Siguiendo a Davidson, supondré que nuestra vida mental se restringe a nuestros estados intencionales. Para una caracterización davidsoniana de "mentalidad", véase Davidson (1970), pp. 210-212.

5 La posición davidsoniana acerca de la causación mental fue expuesta originariamente en Davidson (1970), y ha sido ratificada más tarde en Davidson (1990) "Thinking Causes", reimpreso en Heil y Mele (1993) Mental Causation, Clarendon Press, Oxford. 
(i) el objeto $O$ tiene un peso (expresado en kilogramos) de $n$;

(ii) el individuo $I$ tiene la creencia de que $p .^{6}$

En ambos casos estamos frente a oraciones cuya estructura parece ser semejante: un objeto concreto -un objeto físico o un ser humano, respectivamente- se encuentra en una relación - de peso, o de creencia- con una entidad abstracta - un número en el primer caso, una proposición en el segundo. (i) Es un caso de "actitud numérica", (ii) de "actitud proposicional".

A partir de este simple hecho es posible extraer las siguientes consecuencias para una teoría de la mente:

(a) dado que hay teorías físicas exitosas en las cuales se apela a actitudes numéricas no hay razones para pensar que no es posible formular, en principio, teorías psicológicas exitosas que involucren estados con contenido proposicional, es decir, actitudes proposicionales.

(b) Dado que no tenemos dificultades para aceptar que el peso (de $5 \mathrm{~kg}$ ) del objeto $O$ fue lo que causó que se rompiera la mesa de vidrio sobre la que $O$ estaba apoyado, parece no haber razones, en principio, para no aceptar que, por ejemplo, la creencia (de que $p$ ) del individuo $I$ causó cierta conducta que él realizó. No hay ningún problema especial para la psicología en lo que respecta al papel causal de estados (o eventos, en la terminología de Davidson) que se identifican apelando a entidades abstractas. ${ }^{7}$

Hasta aquí, he mencionado una serie de coincidencias entre las actitudes numéricas y las actitudes proposicionales, a saber:

(1) Se establecen relaciones cuyo sujeto son objetos físicos, en el primer caso y seres humanos, en el segundo; en ambos casos se trata de entidades concretas, situadas en el espacio-tiempo.

6 Tal como está planteado el paralelo hasta aquí, hay una evidente asimetría entre ambos casos, dado que en (i) se ha agregado entre paréntesis la frase "expresado en kilogramos"; y en (ii) no hay nada que se corresponda con ésta, dado que las proposiciones no pertenecen a ningún lenguaje. Sin embargo, la simetría entre los dos casos se vuelve a restaurar si se considera que las entidades con las que el verbo de actitud proposicional pone en relación al individuo no son proposiciones, sino algún tipo de entidad lingüística, como oraciones o emisiones ( $\mathrm{y}$ es esto último lo que sostiene Davidson). Volveré sobre las consecuencias de adoptar una u otra de estas decisiones más adelante, en el apartado [c] punto [2].

7 Ésta es, a mi juicio, la consecuencia que trae Dennett de esta analogía, tal como la desarrolla en "Beyond Belief", reimpreso en Dennett (1987) pp. 123-126. En realidad, la conclusión de Curchland es sustancialmente la misma (aunque no mencione específicamente las relaciones causales), dado que defiende la posibilidad de formular leyes (no vacias) tanto utilizando actitudes numéricas como actitudes proposicionales; véase Churchland (1984) pp. 64-66. 
(2) Del otro lado, la relación se establece con entidades abstractas: números, en un caso; proposiciones, en el otro.

(3) Entre los objetos físicos se dan, efectivamente, ciertas relaciones, como "ser más alto que...", o "estar tan caliente como..."; y en el caso de los seres humanos, relaciones como "pensar lo mismo que...", "desear lo contrario que..." Estas relaciones son las que se pretende reflejar al adoptar un sistema de medición, numérico, en el primer caso; proposicional, en el segundo.

(4) Estas relaciones se pueden poner en correspondencia con ciertas relaciones existentes entre los objetos abstractos que se adoptan para medir los objetos de (1): relaciones como "ser mayor que...", en el caso de los números o relaciones de "implicación lógica y apoyo evidencial", 8 en el caso de las proposiciones.

Sin embargo, la analogía tal como la presenta Davidson es, a mi juicio, mucho más profunda. En lo que sigue, intentaré mostrar que hay una lista adicional de coincidencias entre nuestro hablar acerca de la medida de objetos físicos, y nuestro hablar acerca de las actitudes proposicionales de los seres humanos, coincidencias que permiten iluminar ciertas tesis davidsonianas acerca de lo mental.

\section{[a]}

Una primera coincidencia, podría sostenerse, reside en la finalidad por la cual nos vemos llevados a adoptar ambos lenguajes. En el caso de los objetos físicos macroscópicos adoptamos un sistema de medición para abreviar nuestros informes acerca de ciertos hechos concernientes a los objetos concretos: al decir cuánto pesa un objeto tratamos de informar acerca de la existencia de ciertas relaciones entre objetos (por ejemplo, que unos pesan más que otros, que dos de ellos pesan lo mismo, etc.). Paralelamente, podría sostenerse que adoptamos nuestro hablar en términos de actitudes proposicionales para no perder de vista ciertas propiedades y relaciones relevantes de los estados psicológicos de los individuos. En palabras de Davidson: "cuando hablamos acerca de cuánto pesan las cosas hablamos de una manera relacional: relacionamos objetos con números y, de este modo, unos objetos con otros". 9 Si extendemos al caso de las actitudes proposicionales esta afirmación acerca de lo que permite un sistema de medición, obtenemos la siguiente afirmación: hablar acerca de las creencias es relacional, relaciona personas con proposiciones, y así unas personas con

8 Davidson (1991), p. 158.

9 Ibid., p. 153. 
otras. El lenguaje intencional puede entenderse entonces como una manera abreviada de describir ciertas (complejas) relaciones interpersonales.

Es bien sabido que Davidson ha defendido profusamente una posición externalista, ${ }^{10}$ de acuerdo con la cual nuestros pensamientos están constituidos en parte por factores externos a los individuos que los poseen. Realizan un papel central en la determinación de los pensamientos dos tipos de factores: las relaciones causales con el mundo que rodea al individuo, y los condicionamientos sociales derivados, principalmente, del papel que desempeñan los demás en el aprendizaje del lenguaje. Se podría hacer una lista de una gran cantidad de diferencias entre el externalismo de Davidson y otras formas de externalismo como las de Putnam y Burge. ${ }^{11}$ Sin embargo, hay una diferencia fundamental que me interesa destacar, que es la negativa sistemática de Davidson a adoptar el externalismo como conclusión surgida a partir de un experimento mental y, en cambio, basar su externalismo en hechos corrientes relativos al aprendizaje del lenguaje. Davidson sostiene que los estados mentales se identifican, en parte, apelando a su historia causal, e incluye las relaciones sociales como parte de esa cadena causal. ${ }^{12}$ Y estas relaciones con los demás y con el mundo son las que el lenguaje intencional está llamado a preservar. Así, tanto en el caso de los sistemas de medida de los objetos físicos, como en los sistemas de adscripción de estados mentales a los seres humanos, la apelación a entidades abstractas (números en el primer caso, proposiciones en el segundo) puede entenderse como un mero recurso lingüístico, i.e., como la adopción de un lenguaje más simple para describir relaciones entre objetos concretos (objetos físicos en el primer caso, seres humanos con otros seres humanos y con su entorno en el segundo caso). Volveré sobre estas ideas en [c] y [d].

[b]

Un segundo aspecto en el que la analogía con los sistemas de medida de los objetos macroscópicos permite comprender mejor a Davidson es atendiendo a las consecuencias ontológicas que se derivan de la adopción de uno y

10 Davidson ha defendido el externalismo tanto del significado lingüístico como de los estados intencionales (los "pensamientos") en Davidson (1988a) "El mito de lo subjetivo", Davidson (1988b) "Las condiciones del pensamiento", Davidson (1986) "El conocimiento de la propia mente", todos reimpresos en Davidson (1992) Mente, mundo y acción, Paidós, Barcelona; así como en Davidson (1990) "Epistemología externalizada", Análisis filosófico, vol. X. no. 1.

11 Mencionar exhaustivamente las diferencias entre las distintas versiones del externalismo excede largamente los límites de este trabajo. Algunos de los pasajes en los que el propio Davidson se ocupa de este tema son: Davidson (1990), pp. 5-10 y Davidson (1986), pp. 131141.

12 Davidson (1990), p. 10. 
otro lenguaje (el lenguaje que permite medir objetos físicos, y el lenguaje intencional). En efecto, introducir un patrón de medida u otro no altera las relaciones preexistentes entre los objetos ni nos obliga a agregar nuevas entidades a nuestra ontología; en palabras de Davidson: "no nos compromete con los pesos como objetos". ${ }^{13}$ Es sólo una manera de relacionar un objeto con otros a los que se les aplica una misma escala. De la misma manera:

cuando se piensa y habla acerca de las creencias de las personas no necesitamos suponer que existan entidades tales como las creencias. Ni tampoco tenemos que inventar objetos que sirvan como los "objetos de creencia" o como lo que está ante la mente, o en el cerebro. ${ }^{14}$

En este punto, Davidson parece coincidir con la estrategia de Dennett, quien muestra cómo es posible adoptar un hablar acerca de actitudes proposicionales, concibiéndolas como relaciones de un individuo con una proposición, y aceptando que tales actitudes cumplen un papel causal sin que por ello debamos suponer que hay algo concreto en los cerebros de los individuos (por ejemplo, oraciones-caso del "lenguaje del pensamiento") que intervenga en la historia causal en la que está involucrada la actitud proposicional, ni tampoco peculiares intermediarios epistémicos o psicológicos como los "objetos ante la mente" postulados por los empiristas modernos. ${ }^{15}$

La analogía con las medidas de los objetos físicos abre un espacio para que la afirmación davidsoniana de la inexistencia de objetos ante la mente ${ }^{16}$ resulte sostenible. Los números no son intrínsecos ${ }^{17}$ a los objetos físicos que tienen peso, ni son parte de ellos, en la medida en que, como se dijo en [a], se trata simplemente de una manera de informar acerca de la existencia de ciertas relaciones entre objetos concretos. De la misma manera, se puede

13 Davidson (1991), p. 153.

14 Ibid., p. 154.

15 Los "objetos ante la mente" u "objetos del pensamiento" a los que alude Davidson son "aquellas entidades fantasmales contempladas de algún modo por la mente" (Davidson (1988b), p. 71). Davidson rechaza tanto la existencia de objetos del pensamiento concebidos como experiencias, sensaciones o datos de los sentidos no interpretados, como si se los concibe como proposiciones plenamente interpretadas, "proposiciones, casos de proposiciones, representaciones o fragmentos de 'mentalés'" (Davidson (1986), p. 148; la misma distinción se encuentra en Davidson (1988b), pp. 62 y 70-71). Dado que en este trabajo me estoy ocupando de las actitudes proposicionales, en lo que sigue consideraré exclusivamente la tesis de la inexistencia del segundo tipo de objetos ante la mente distinguidos por Davidson.

16 Argumentos explícitos en favor de esta tesis pueden encontrarse en Davidson (1988b), (1991).

17 En este apartado, entiendo "intrínseco" como sinónimo de "interno" (y, consecuentemente, como opuesto a "externo"); más adelante este término adquirirá otros dos significados, "intrínseco" como "constitutivo", e "intrínseco" como opuesto a "extrinseco" o "relacional", que se aclararán oportunamente. 
sostener que nuestro hablar en términos de actitudes proposicionales no nos compromete con la existencia de entidades intrínsecas a las personas. La analogía que nos ocupa muestra que es posible separar dos problemas: el problema lógico-semántico relativo al análisis que debemos adoptar para las oraciales de adscripción de actitudes proposicionales, y el problema metafísico acerca de la naturaleza de las actitudes proposicionales mismas; a saber, si -metafísicamente hablando-resultan ser estados relacionales (diádicos o poliádicos) o, por el contrario, se trata de estados no relacionales. ${ }^{18}$ Este divorcio le permite a Davidson adoptar un análisis de las oraciones de adscripción de estados mentales como relaciones entre individuos y emisiones, sin por ello comprometerse con la tesis metafísica de que los estados de actitud proposicional son relaciones diádicas entre un individuo y alguna otra entidad.

\section{[c]}

Hasta aquí he argumentado que el lenguaje de las actitudes proposicionales no nos compromete con la existencia de entidades intrínsecas (i.e., internas) a los objetos de los que se las predica. Sin embargo, T. Crane ${ }^{19}$ ha argumentado que las proposiciones sí son intrínsecas ${ }^{20}$ a la actitud proposicional misma, puesto que la proposición involucrada en una actitud proposicional es esencial para la individuación de esta última. En este aspecto parece surgir una diferencia importante que amenaza la analogía entre el sistema de las actitudes numéricas y el de las actitudes proposicionales. $\mathrm{El}$ argumento de Crane tiene la forma de un dilema. El primer cuerno del dilema consiste en sostener que la analogía entre los sistemas de medición de objetos físicos y las atribuciones de actitudes proposicionales tiene por finalidad mostrar que en ambos casos se establecen genuinas relaciones entre objetos concretos y entidades abstractas. Sin embargo, ésta no puede ser la finalidad de la analogía porque, sostiene Crane, en lugar de resolver el problema de la intencionalidad, traslada a la física el problema de cómo explicar el poder causal de entidades relacionadas con objetos abstractos. El segundo cuerno del dilema afirma que la lectura adecuada de la analogía debe ser que en ninguno de los dos casos se trata de una relación genuina sino que, por el contrario, se utiliza una relación con una entidad abstracta

18 En la introducción de Valdés (1996) se puede encontrar una distinción entre las actitudes propocionales como objeto de estudio de la filosofía de la mente, y las oraciones de atribución de actitudes proposcionales como objeto de estudio de una filosofía del lenguaje (pp. 6-7). Este trabajo está centrado en la discusión del primero de estos dos problemas.

19 T. Crane, "An Alleged Analogy between Numbers and Propositions", Analysis, vol. 50, no. 4, oct. 1990.

20 En este punto, "intrínsecas" debe entenderse en el sentido de "constitutivas". 
para individualizar algo que en realidad es una propiedad intrínseca ${ }^{21}$ del objeto concreto. Así, decir que el objeto $O$ pesa cinco kilos es una manera de hacer referencia a cierta propiedad intrínseca de $O$, y, de la misma manera, decir que $I$ cree que llueve es una manera de hacer referencia a una propiedad intrínseca de $I$. Pero, sigue Crane, de ninguna manera puede aceptarse que las actitudes proposicionales sean estados intrínsecos de los individuos. Un síntoma de este hecho es que si bien es arbitrario qué número se elija para individuar la temperatura entendida como una propiedad intrínseca de un objeto, ya que adoptando distintas unidades de medida se pueden seleccionar distintos números, en el caso de las creencias la proposición involucrada es defintoria de la creencia y no es relativa a una unidad de medida. El argumento, tal como ha sido reconstruido hasta aquí, podría considerarse como una falacia de equívoco, en la medida en que utiliza en sus premisas "intrínseco" en dos sentidos diferentes (como se mencionó en las dos últimas notas). Sin embargo, podría reescribirse la conclusión de su argumento en los siguientes términos: las proposiciones son intrínsecas (en el sentido de constitutivas) de las actitudes proposicionales, por lo tanto las actitudes proposicionales no pueden ser propiedades intrínsecas (en el sentido de no-relacionales) de los individuos. Adopto esta lectura del argumento de Crane, por lo que, en lo que sigue, me detendré en el problema de si las actitudes proposicionales son propiedades intrínsecas (en el sentido de no-relacionales) de los individuos, o no. El argumento de Crane merece, en mi opinión, los siguientes dos comentarios:

[1] Si uno relee los términos en los cuales Davidson plantea la analogía mencionados en [a] y [b], se puede dar una lectura diferente de la analogía, que Crane no considera. La analogía, como se ha dicho, muestra el divorcio existente entre el problema semántico relativo al análisis adecuado de las adscripciones de actitudes proposicionales, y el problema metafísico relativo a la naturaleza de dichas actitudes. Crane considera sólo dos tipos de respuesta posibles a este último problema. Sostiene que o bien (i) se trata de relaciones genuinas entre un objeto concreto y una entidad abstracta, o bien (ii) se trata de la afirmación de una propiedad intrínseca de los objetos considerados. Yo propongo una tercera respuesta para el problema metafísico: (iii) lo que en realidad se afirma es una relación nádica que involucra al objeto concreto y a todos los otros objetos a los que se les puede aplicar la misma escala de medidá (así como a los objetos del entorno compartido). Es algo así como una propiedad super-relacional en tanto relaciona a un individuo con sus semejantes y con su medio. En

21 En este paso del argumento de Crane, "intrínseca" aparece como sinónimo de "norelacional". 
principio, es posible establecer este tipo de relación sin ni siquiera preguntarse si tienen efectivamente alguna propiedad intrínseca en común o no todos aquellos individuos a los que, aplicado un patrón de medida, se les asigna un mismo número. Parece aceptable sostener que tener 1.20 metros de altura por parte de una silla y tener 1.20 metros de estatura por parte de un niño no involucra ninguna propiedad intrínseca que esa silla y ese niño compartan. Lo único que tienen en común es que, aplicada una escala, se les asigna un mismo número. Nótese que, sea cual fuere la escala de medida adoptada, siempre se asignará un mismo número a ambos objetos, porque todos los sistemas de medición están creados para preservar la re'lación "mismo alto que" existente entre el niño y la silla. La asignación de una medida determinada a un objeto concreto, así como la atribución de una actitud proposicional a un individuo dado en un momento determinado no es totalmente arbitraria y caprichosa; está limitada por las otras atribuciones de longitud que se efectúan en el primer caso, y por las demás atribuciones de actitudes proposicionales (y de significados a las emisiones lingüísticas, y de acciones intencionales) que uno está dispuesto a aceptar para ese individuo y sus semejantes, en circunstancias similares. ${ }^{22}$

Esta tercera respuesta es consistente con mi lectura de la finalidad que Davidson reconoce para adoptar el lenguje de las actitudes proposicionales mencionada en el punto [a]. Y además, esta tercera lectura de la analogía, de motivación nominalista en el caso de los números (ya que permite eliminar el compromiso con entidades abstractas en favor de relaciones, más o menos complejas, entre objetos concretos), es compatible con una lectura nominalista de Davidson, ${ }^{23}$ es decir, con una lectura de Davidson de acuerdo con la cual su intento es también, después de todo, deshacerse de ciertas entidades abstractas, a saber, las proposiciones. Esto me lleva a mi segunda observación al argumento de Crane.

22 Recuérdese el holismo de lo intencional, asumido por Davidson a lo largo de toda su obra. Algunos de los lugares en los que menciona Davidson esta tesis son: Davidson (1970), pp. 217 y 221-223; Davidson (1973) "The Material Mind", p. 257 y Davidson (1974a) "Psychology as Philosophy", p. 231, ambos reimpresos en Davidson (1980); Davidson (1974b) "Belief and the Basis of Meaning", Synthèse, 27, p. 322; Davidson (1986), pp. 137-138; Davidson (1990), p. 3; y Davidson (1991), p. 160.

23 Hay varios sentidos en los que se puede decir que Davidson es nominalista. Uno es el mencionado en el cuerpo de este trabajo. Un segundo sentido surge de la lectura de Davidson (1970) donde al describir su monismo anómalo se compromete sólo con (i) eventos concretos particulares, y (ii) descripciones (lingüísticas) de los eventos, pretendiendo que sólo con particulares concretos y expresiones lingüísticas alcanza para dar cuenta de nuestra vida mental. Esta posible lectura nominalista de Davidson (1970) fue señalada por la profesora Cristina González en el curso del seminario sobre la filosofía de Davidson mencionado en la última nota de este trabajo. 
[2] La analogía de los sistemas de medida con las atribuciones de actitudes proposicionales puede proponerse con dos finalidades diferentes: (i) para defender la idea de que concebir las actitudes proposicionales como relaciones con entidades abstractas no genera problemas especiales a la psicología; ${ }^{24}$ o bien (ii) para defender la idea de que el carácter relacional de la expresión lingüística "cree" no causa problemas a la psicología. Creo que la actitud de Davidson es del segundo tipo. Y por ello, después de haber discutido extensamente la analogía en "What is Present to the Mind?", reconoce no haberse pronunciado todavía acerca de cuáles son los objetos que se utilizan para individuar el estado de una persona cuando se le aplica la palabra relacional "cree". ${ }^{25} \mathrm{Y}$ en este punto se pronuncia por las emisiones" como el tipo de entidad con la cual el verbo de actitud proposicional pone en relación a los individuos. Nos dice:

Si Ud. lo desea, hasta podría considerar que el objeto es una proposición. Dado que las emisiones, las oraciones y las proposiciones están tan íntimamente relacionadas, es probable que si una elección funciona, pueda hacerse que las otras también funcionen. Pero las emisiones tienen ciertas ventajas prima facie, ya que no son abstractas, y por eso están atadas a un hablante, un tiempo y un contexto. Asumiré, pues, que hemos escogido las emisiones, las mismas emisiones que se producen al atribuir actitudes, como los objetos que sirven para individuar e identificar los diversos estados de la mente. ${ }^{26}$

Así, la analogía propuesta es importante para defender el carácter relacional de "cree" y, consecuentemente, el punto de Crane no sirve como crítica a la propuesta davidsoniana, porque desde este punto de vista se niega que sean las proposiciones involucradas en las atribuciones de creencias las que identifiquen tales creencias. Son las emisiones de los que atribuyen las actitudes proposicionales las que permiten individuar las creencias. Toda emisión pertenece a uno u otro lenguaje, por lo que la individuación de las creencias se vuelve relativa a unidades o a patrones, esto es al lenguaje usado por el intérprete, y así las proposiciones no resultan constitutivas de las actitudes proposicionales. Si se diera lo contrario, habría una manera independiente de todo lenguaje de determinar los estados mentales en los que se encuentran los individuos; pero esto, dada la estrecha relación pos-

24 En este punto, por "psicología" no me refiero a una disciplina científica perteneciente al ámbito de la ciencia natural, sino a algo así como una teoría de lo intencional, ya que, como es sabido, al negar la existencia de leyes estrictas que involucren términos intencionales, Davidson excluye la psicolgía del ámbito de las ciencias naturales.

25 Davidson (1991), p. 157.

26 Ibid., p. 158. 
tulada por Davidson entre pensamiento y lenguaje $\mathrm{e}^{27}$ es inconcebible en el marco de su filosofía. Veamos qué consecuencias tiene esto.

\section{[d]}

De la misma manera que es posible asignar distintos números para la tarea única de medir el peso de los objetos físicos (por ejemplo se asignarán distintos números a un mismo objeto si se expresa su peso en libras y en kilogramos, o aún en kilogramos y en gramos), también es posible apelar a distintas emisiones para describir la misma creencia de un individuo. ${ }^{28}$ Esto ocurre, por ejemplo si dos hablantes de distintos lenguajes naturales (digamos, español e inglés) describen las creencias de un tercer individuo en un momento dado. Pero, aclara Davidson, del hecho de que se usen distintos conjuntos de números para medir el peso de un cuerpo, no se deduce que el peso no sea algo "real", ni que la asignación de peso sea "relativista". Del mismo modo, las emisiones de una persona pueden captar todos los rasgos significativos del pensamiento de otra persona de distintas maneras. Puede haber más de un conjunto de emisiones que resulten igualmente exitosas para capturar los contenidos de la mente de una persona. Pero esto no amenaza la realidad de las actitudes. Si uno niega, como hace Davidson, que al atribuir una creencia uno esté identificando un objeto con el cual el sujeto está en una relación peculiar de "captación" [grasping], no es necesario elegir una de entre muchas interpretaciones exitosas como el contenido de la creencia. ${ }^{29}$ Esto es así, porque, como se dijo en [b], al adoptar un patrón de medida no se agregan objetos a la ontología ni se alteran las relaciones preexistentes entre los individuos; y además porque, como he propuesto en el punto [2] del apartado [c], la lectura adecuada de Davidson consiste en pensar las atribuciones de creencias como super-relacionales, estableciendo

27 Davidson sostiene que "ni el lenguaje ni el pensamiento pueden explicarse completamente en términos del otro, y ninguno de los dos tiene prioridad conceptual. Los dos, por cierto, están relacionados, en el sentido de que cada uno requiere del otro para su comprensión; pero la relación no es tan completa como para que alguno de ambos baste, incluso cuando se lo refuerce razonablemente, para explicar al otro", en Davidson (1975) "Talk and Thought", Mind and Language, Guttenplan (comp.), Oxford University Press, p. 8.

28 Con esto se anula la única diferencia visible en la analogía original, que se había mencionado en la n. 6.

29 Esto explica, además, por qué las atribuciones en primera persona no son infalibles. Las atribuciones de estados mentales que hago de mí misma son unas de entre las que hacen otros intérpretes. Puedo hacerlas por medio de una emisión externa (en voz alta) o interna (cuando me digo a mi misma que estoy creyendo que $p$ o deseando que $q$ ). Y las atribuciones que yo me hago serán correctas o no, dependiendo de exactamente los mismos parámetros con los que se juzgan las atribuciones que me hacen otros intérpretes. $O$ todas son falibles, o ninguna lo es. 
relaciones entre unos individuos y otros, y con el entorno, y no con objetos a los que tendríamos un oscuro modo de acceso. Podemos usar distintos conjuntos de emisiones para describir el mismo conjunto de relaciones interpersonales. Similitudes, observadas o supuestas, entre diferentes estados en los que los humanos nos relacionamos unos con otros y con nuestro entorno, quedan reflejadas por medio de las emisiones con las que identificamos los estados mentales de los humanos involucrados, pero dichas emisiones no constituyen los estados mentales en cuestión, sólo resultan herramientas útiles para señalar similitudes objetivas entre diversos estados relacionales complejos. Pensar que no es posible adoptar diversos conjuntos de emisiones para referirnos al mismo conjunto de similitudes observadas sería como pensar que "la 'diferencia' entre tener una yarda de largo y tener una longitud de 36 pulgadas al medir con una yarda patrón fuese una diferencia en la yarda patrón misma". ${ }^{30}$

Recordemos, además, que Davidson pretende haber establecido la imposibilidad de una formulación clara de la tesis relativista. ${ }^{31}$ Y que más tarde, en "El mito de lo subjetivo", defiende la idea de que si bien las creencias de un individuo cualquiera se expresan en uno u otro lenguaje natural, esto no nos compromete con la idea de que las creencias sean relativas a esquemas, porque son las representaciones las que resultan relativas a esquemas y al adscribir a alguien una creencia no nos hemos de comprometer con una "representación mental", es decir, con un "objeto ante la mente".

Resumiendo, hasta aquí he intentado mostrar que la analogía entre los sistemas de medición de objetos físicos y los sistemas de atribución de actitudes proposicionales a los seres humanos le permite a Davidson mostrar cómo la tesis de la relacionalidad de "cree", que él defiende, no lo compromete con una tesis metafísica respecto de la naturaleza de los estados mentales de acuerdo con la cual ellos son vistos como estados relacionales diádicos entre un individuo y una entidad determinada, sea una proposición, un "objeto ante la mente" o una inscripción "en la cabeza". Por el contrario, Davidson rechaza estas concepciones de lo mental y acepta que la relación que "cree" establece (con una emisión lingüística de un intérprete), permite individuar cierto estado relacional complejo en el que se destacan ciertas similitudes objetivas existentes entre aquel al que se le atribuye el estado mental, los demás seres humanos, y los objetos del mundo exterior observados por ambos.

En los próximos apartados me detendré en otros dos aspectos de la filosofía de la mente davidsoniana: el tema de la racionalidad como principio

30 Davidson (1991), p. 160.

31 Davidson (1974) "On The Very Idea of a Conceptual Scheme", reimpreso en Davidson (1984). 
constitutivo de lo mental y el problema de la causación mental. Intentaré mostrar en qué medida la analogía que es tema de este artículo permite (o no) echar luz sobre estos dos asuntos.

\section{[e]}

En su famosísimo "Mental Events" también propone Davidson una analogía entre los sistemas de medición de objetos físicos macroscópicos y los sistemas de conceptos mentales. ${ }^{32}$ Sin embargo, el contexto en el cual aparece esta analogía es bien diferente. Davidson se propone en la parte II de ese artículo probar que no hay leyes psicofísicas. El esqueleto de este argumento es el siguiente: lo mental y lo físico constituyen dos sistemas de conceptos diferentes, en el sentido de que cada uno de estos sistemas posee principios constitutivos diferentes. Dos ámbitos que poseen principios constitutivos radicalmente diferentes no pueden hallarse en una conexión estrecha como la que requiere una conexión nómica, esto es, como la que se establece cuando se formula una ley uniendo ambos conceptos. Por lo tanto, no hay leyes psicofísicas. ${ }^{33}$ Dos puntos quedan pendientes en este argumento. Primero, explicar qué se entiende por "principios constitutivos" de un sistema de conceptos. ${ }^{34}$ Segundo, la explicitación de los principios constitutivos de los dos ámbitos de conceptos con los que Davidson está tratando en este caso: lo mental y lo físico.

Es en cuanto al primero de estos puntos que la analogía con los sistemas de medición resulta particularmente importante. Porque es precisamente al tratar de aclarar qué se entiende por "principios constitutivos" de una teoría que Davidson introduce la analogía con los sistemas de medición. Siguiendo a Davidson, cuando nos enfrentamos con el conjunto de las leyes y postulados que rigen la medición de los objetos físicos macroscópicos no tiene sentido preguntarse si cada una de esas leyes o axiomas son analíticos o sintéticos, es decir, si son verdaderos en virtud del significado de los términos

32 Davidson usa en su (1970) la expresión "conceptos mentales" para referirse a aquellas descripciones de los eventos que involucran términos mentalistas. Por lo tanto, los "conceptos mentales" son, para Davidson, entidades lingüísticas. Respeto a lo largo de este apartado la terminología davidsoniana, por lo tanto la naturaleza lingüística de los conceptos debe tenerse presente a lo largo de [e].

33 Para una excelente y detallada exposición del argumento davidsoniano en contra de las leyes psicofísicas, puede consultarse Kim (1985) "Psychophysical Laws", reimpreso en Kim (1993) Supervenience and Mind, Cambridge University Press, Cambridge.

34 Recordemos que, para Davidson, los conceptos mentales son entidades lingüísticas (como se señaló en la n. 32), que las leyes también son entidades lingüísticas, y que los eventos son mentales o físicos en virtud de las descripciones que se den de ellos, por lo tanto, los "principios constitutivos" de lo físico y de lo mental, serán determinadas relaciones que se establezcan entre entidades lingüísticas. 
o si lo son por razones empíricas. Davidson adopta explícitamente la tesis quineana de acuerdo con la cual hay que rechazar una distinción tajante entre enunciados analíticos y sintéticos. Pero entonces, ¿qué estatus tienen, desde esta perspectiva, los postulados y leyes de la teoría de la medición? La respuesta de Davidson es: son leyes constitutivas, es decir, sintéticas a priori. En sus propias palabras: "el conjunto total de axiomas, leyes, o postulados para la medida de longitud es parte constitutiva de la idea de un sistema de objetos macroscópicos, rígidos, físicos". ${ }^{35}$ Esto quiere decir que el hecho de que un objeto deba tener algún largo, algún ancho y algún fondo es parte constitutiva de nuestro concepto de objeto físico. Paralelamente, en el caso del sistema de conceptos mentales, Davidson también considera que hay ciertos principios que lo rigen. Así, sostiene que "no podemos atribuir ninguna actitud proposicional a un agente excepto dentro del marco de una teoría viable de sus creencias, deseos, intenciones y decisiones", ${ }^{36}$ y este marco de creencias y deseos está gobernado por "el ideal constitutivo de la racionalidad".37

De esta manera, la analogía entre los sistemas de medición de objetos físicos y los conceptos mentales sirve, en esta oportunidad, para comprender el papel que desempeña la racionalidad dentro de la filosofía de la mente de Davidson. La racionalidad es un principio constitutivo de lo mental, esto es, de lo intencional. Abandonar la racionalidad de lo mental -es decir, fundamentalmente de las creencias, deseos y acciones humanas-, sería como "cambiar de tema", es decir, no aceptar como criterio de lo mental el vocabulario de las actitudes proposicionales, y, consecuentemente, abandonar nuestra concepción del hombre como animal racional.

En cuanto al segundo de los puntos pendientes, esto es cuáles son efectivamente los principios constitutivos que gobiernan el ámbito de lo físico y el ámbito de lo mental, la analogía que estamos investigando no nos ayuda, a mi juicio, a encontrar una respuesta. Sin embargo, la analogía señala el camino que hay que seguir para determinar cuáles son los principios constitutivos de cada uno de estos ámbitos: en el caso de lo físico hay que atender a los axiomas y postulados de la ciencia física, en el caso de lo mental el problema de la racionalidad se resuelve recurriendo a las teorías existentes que buscan sistematizar las nociones de acción racional, creencia y preferencia, es decir, a la teoría de la decisión.

En síntesis, en lo que respecta al tema de la racionalidad en la filosofía de la mente de Davidson, la analogía entre los sistemas de medición de objetos físicos macroscópicos y los sistemas de conceptos mentales permite

37 Ibid., p. 223. 
tener una mejor comprensión del papel que ésta desempeña en su teoría, a saber, el de principio constitutivo de lo mental; pero no nos ayuda a la explicitación de en qué consiste tal racionalidad, ni en el problema de qué otros principios constitutivos de lo mental interactuarían (si los hubiere) con la racionalidad para gobernar nuestros conceptos mentales.

\section{[f]}

Finalmente, unas palabras acerca del problema que ha llevado a popularizar la analogía entre los sistemas de medición de objetos físicos y el lenguaje en términos de actitudes proposicionales en la literatura filosófica de los últimos años: el problema de la causación mental. La respuesta davidsoniana a este problema se hallaba expuesta ya en "Mental Events": los eventos mentales y los eventos físicos (en tanto entidades particulares, concretas, fechables) entran en relaciones causales. Estas conexiones causales son relaciones diádicas extensionales entre eventos particulares. ${ }^{38}$ Las leyes causales, por el contrario, no involucran directamente a los eventos particulares sino a través de las diferentes descripciones que de ellos es posible hacer. De esta manera, resulta posible conciliar la idea de que los eventos mentales y los físicos entran en relaciones causales, aunque no sea posible establecer leyes psicofísicas entre ellos: para todo evento mental habrá una descripción física por medio de la cual se puedan establecer leyes estrictas en las que tal evento se encuentre involucrado. Dado que los eventos davidsonianos son particulares concretos, son los mismos eventos los que pueden ser descritos física o mentalmente. ${ }^{39}$ Éste es, a grandes trazos, el "monismo anómalo" de Davidson. ${ }^{40}$

Ahora bien, ¿es compatible esta teoría con la adopción de la analogía entre sistemas de medición de objetos físicos macroscópicos y los sistemas de conceptos mentales? La respuesta es la siguiente. Esta analogía como se dijo más arriba, nos ayuda a distinguir dos planos: el semántico y el ontológico. Desde el punto de vista semántico, las oraciones mediante

38 Este tema está desarrollado, además de en Davidson (1970), en Davidson (1967), "Causal Relations", reimpreso en Davidson (1980).

39 Esta tesis se conoce con el nombre de teoría de la identidad de casos, y en su (1970) Davidson propone un argumento en su favor.

40 Para una exposición más detallada del monismo anómalo de Davidson y mi evaluación de su respuesta al problema de la impotencia causal de lo mental puede verse mi "Monismo anómalo y causación psicofísica", Análisis filosófico, vol. XIV, no. 1, mayo de 1994. La respuesta davidsoniana a este problema depende de su peculiar concepción de la causación como una relación diádica extensional entre eventos particulares. Este análisis de la causación es, desde mi perspectiva, inadecuado, y es por esa razón que la respuesta davidsoniana al problema de la causación mental me parece inaceptable. Desarrollo con detalle este punto de vista en el artículo mencionado y en mi tesis doctoral: La mente como eslabón causal (inédita). 
las cuales adscribimos actitudes proposicionales son relacionales, pero ello no debe comprometernos con una ontología relacional. ${ }^{41}$ En otros términos, pensar las actitudes proposicionales como relaciones con proposiciones $(\mathrm{u}$ oraciones, o emisiones) nos permite contar con una manera de identificar ciertas entidades (en el caso que nos ocupa, eventos mentales), pero no nos compromete con una respuesta unívoca al problema de la naturaleza de las entidades identificadas de esa manera. $\mathrm{O}$ aún en otros términos, todo sistema de medición supone una respuesta al problerna de la representación, es decir, una especificación de un conjunto de propiedades y relaciones empíricas que se preservan por medio de una función que se establece entre ellas y el conjunto de entidades (números, proposiciones) que constituyen la escala utilizada para efectuar la medición de los objetos físicos. Pero el sistema de medición, por sí mismo, nada dice respecto de cuáles sean las propiedades y relaciones que se busca preservar a través de un determinado sistema de medición. Tal vez, como sugiere Matthews, sea un problema empírico el de determinar cuáles son las propiedades y relaciones que nuestros sistemas de actitudes proposicionales están destinados a preservar. ${ }^{42}$ En cualquier caso, lo importante a destacar es lo siguiente: ningún sistema de medidas altera las propiedades y relaciones existentes entre los objetos medidos, por lo tanto, en el caso de los eventos mentales, si estos efectivamente entran en relaciones causales, tal como Davidson afirma, identificarlos por medio de una descripción física, o por medio del sistema de las actitudes proposicionales no altera en lo más mínimo las relaciones causales existentes entre los eventos que conforman el mundo. ${ }^{43}$

41 Algunas de las ontologías alternativas, compatibles con la tesis semántica de la relacionalidad, fueron mencionadas en (i)-(iii), apartado [c] punto [2].

42 Matthews (1994) "The Measure of Mind", Mind, vol. 103, p. 140. Si bien Matthews considera que es un problema empírico el de determinar qué propiedades y relaciones preserva el lenguaje de las actitudes proposicionales sostiene que "las propiedades y relaciones [recogidas por el lenguaje de las actitudes proposicionales] son aquellas propiedades causalmente eficaces de los estados internos" (p. 139, en la página 144 hace una afirmación similar en el mismo sentido). Por esta razón, en mi opinión, se ubicaría a sí mismo en la opción (ii) de las mencionadas más arriba en el apartado [c].

43 La versión definitiva de este trabajo se ha visto enriquecida gracias a comentarios y discusiones mantenidas con Horacio Arlo, Fernando Broncano, Antoni Gomila, Sandra Lazzer, Eduardo Rabossi, Marcelo Sabatés, Tomás Simpson, Liza Skidelsky, David Sosa y Margarita Vázquez, así como a las discusiones producidas en el ámbito de los seminarios sobre Actitudes proposicionales (coordinado por T. Simpson, SADAF, 1993) y sobre la Filosofía de D. Davidson (coordinado por E. Rabossi y A. Moretti, SADAF, 1994). Una versión anterior de este trabajo fue leída en el Segundo Coloquio de Filosofía de Bariloche (30 de junio y el 2 de julio de 1994). Agradezco asimismo los valiosos y extensos comentarios realizados por el dictaminador anónimo de Diánoia que me han permitido mejorar sustancialmente la versión definitiva de este trabajo. 